\title{
Iron supplementation promotes gut microbiota metabolic activity but not colitis markers in human gut microbiota-associated rats
}

\author{
Alexandra Dostal ${ }^{1}$, Christophe Lacroix ${ }^{1 *}$, Van T. Pham ${ }^{1}$, Michael B. Zimmermann ${ }^{2}$, \\ Christophe Del'homme ${ }^{3}$, Annick Bernalier-Donadille ${ }^{3}$ and Christophe Chassard ${ }^{1}$ \\ ${ }^{1}$ Laboratory of Food Biotechnology, Institute of Food, Nutrition and Health, ETH Zurich, Switzerland \\ ${ }^{2}$ Laboratory of Human Nutrition, Institute of Food, Nutrition and Health, ETH Zurich, Switzerland \\ ${ }^{3}$ UR 454 Microbiology Unit, INRA, Clermont-Ferrand Research Centre, St Genès-Champanelle, France
}

(Submitted 17 June 2013 - Final revision received 14 November 2013 - Accepted 14 January 2014 - First published online 21 February 2014)

\section{Abstract}

The global prevalence of Fe deficiency is high and a common corrective strategy is oral Fe supplementation, which may affect the commensal gut microbiota and gastrointestinal health. The aim of the present study was to investigate the impact of different dietary Fe concentrations on the gut microbiota and gut health of rats inoculated with human faecal microbiota. Rats ( 8 weeks old, $n$ 40) were divided into five ( $n 8$ each) groups and fed diets differing only in Fe concentration during an Fe-depletion period (12 weeks) and an Fe-repletion period ( 4 weeks) as follows: (1) Fe-sufficient diet throughout the study period; (2) Fe-sufficient diet followed by 70 mg $\mathrm{Fe} / \mathrm{kg}$ diet; (3) Fe-depleted diet throughout the study period; (4) Fe-depleted diet followed by $35 \mathrm{mg} \mathrm{Fe} / \mathrm{kg}$ diet; (5) Fe-depleted diet followed by $70 \mathrm{mg} \mathrm{Fe} / \mathrm{kg}$ diet. Faecal and caecal samples were analysed for gut microbiota composition (quantitative PCR and pyrosequencing) and bacterial metabolites (HPLC), and intestinal tissue samples were investigated histologically. Fe depletion did not significantly alter dominant populations of the gut microbiota and did not induce Fe-deficiency anaemia in the studied rats. Provision of the $35 \mathrm{mg}$ Fe/kg diet after feeding an Fe-deficient diet significantly increased the abundance of dominant bacterial groups such as Bacteroides spp. and Clostridium cluster IV members compared with that of an Fe-deficient diet. Fe supplementation increased gut microbial butyrate concentration 6-fold compared with Fe depletion and did not affect histological colitis scores. The present results suggest that Fe supplementation enhances the concentration of beneficial gut microbiota metabolites and thus may contribute to gut health.

Key words: Iron: Gut microbiota: Colitis: Butyrate

Research carried out in the past few years has highlighted the importance of the gut microbiota to intestinal health and host health in general $^{(1,2)}$. The gut microbiota plays a crucial role in the development and maintenance of the immune system ${ }^{(3)}$, colonisation resistance to environmental bacteria, such as pathogens $^{(4)}$, extraction of energy from indigestible food components and production of metabolites that influence gut health ${ }^{(5)}$. SCFA have been widely investigated due to their ability to influence several aspects of host health. Many members of the gut microbiota produce acetate and propionate. Acetate is either reused by other bacteria for butyrate formation or absorbed by the host and can be used as an energy source, while propionate is involved in gluconeogenesis $^{(6,7)}$. The most investigated end metabolite of the gut microbiota is probably butyrate due to its importance to mucosal health. Butyrate is mainly produced by the members of Clostridium clusters IV and XIVa, such as Faecalibacterium prausnitzii, Eubacterium hallii and Roseburia spp., ${ }^{(8,9)}$ and is absorbed by the mucosa where it can act as an energy source for colonocytes ${ }^{(10)}$. Moreover, butyrate has anti-inflammatory effects, is able to promote apoptosis in cancer cells and has been shown to inhibit virulence in enteropathogens ${ }^{(11,12)}$.

Gut microbiota composition and metabolic activity not only affect the host but are also strongly modified by host and environmental factors. Diet composition, such as fibre content, has been shown to have a strong impact on the gut microbiota ${ }^{(13,14)}$. Recent studies have elucidated the differences in gut microbial consortium in populations living in developed and developing countries possibly due to the effects of diet or high prevalence of malnutrition in developing countries, which can strongly influence the gut microbiota $^{(3,15-20)}$. One of the most common nutritional deficiencies is Fe deficiency, affecting more than two billion people worldwide, especially in developing countries ${ }^{(21,22)}$

Abbreviations: qPCR, quantitative PCR; SRB, sulphate-reducing bacteria.

*Corresponding author: C. Lacroix, fax +41 4463214 03, email christophe.lacroix@hest.ethz.ch 
Fe deficiency affects cognitive and motor development in children and increases the maternal/perinatal mortality rate $^{(23)}$. It also has an impact on gut microbiota composition and metabolic activity. In a recent rat study, we have shown a decrease in the abundance of Roseburia spp./Eubacterium rectale and Bacteroides spp. along with a large decrease in the concentrations of caecal butyrate and propionate in highly Fe-deficient young rats $^{(24)}$. Earlier studies in mice have also demonstrated that low gut luminal Fe concentrations have an impact on gut microbiota composition leading to a decrease in the abundance of Desulfovibrio spp. and an increase in that of Turicibacter spp. ${ }^{(25)}$ while elevating the counts of lactobacilli ${ }^{(26)}$. Using an in vitro colonic fermentation model mimicking the proximal colon of a child, we have also confirmed strong dysbiosis of the gut microbiota under very-low-Fe conditions ${ }^{(27)}$.

Usual strategies for Fe-deficiency anaemia correction are Fe supplementation and Fe fortification of foods; $\mathrm{FeSO}_{4}$ is often used, which is highly bioavailable ${ }^{(28)}$. However, absorption of Fe is usually low (5-20\%) and takes place mainly in the duodenum, while the main fraction of $\mathrm{Fe}$ reaches the colon, where it might affect the gut microbiota ${ }^{(21)}$. Indeed, gut microbiota composition in Fe-deficient rats supplemented with $\mathrm{Fe}$ has been found to be partially recovered and gut microbiota metabolic activity to be strongly promoted ${ }^{(24)}$. Animal studies in rats and pigs have also reported dysbiosis of the gut microbiota due to Fe supplementation ${ }^{(26,29)}$, recently confirmed in human studies. School children in Côte d'Ivoire have been found to have higher numbers of Enterobacteriaceae and decreased numbers of lactobacilli in faeces after Fe supplementation for 6 months and increased concentrations of calprotectin, a marker for gut inflammation (30). Infants given an Fe-supplemented diet for 3 months have been found to have higher relative abundances of Bacteroides spp. and lower abundances of lactobacilli ${ }^{(31)}$.

An unanswered question raised by these studies is whether dietary Fe alone is a modulation factor for the gut microbiota and intestinal inflammation or whether Fe status and susceptibility to gut inflammation could also play a role in the observed changes in gut microbiota composition. Both changes in host Fe homeostasis and gut inflammation can alter the gut microbiota composition ${ }^{(32-34)}$. Our previous study using Sprague-Dawley rats with a rodent microbiota has investigated the changes in gut microbiota composition due to Fe supplementation in a highly Fe-deficient host ${ }^{(24)}$. As the rodent microbiota differs from the human microbiota $^{(35-37)}$, in the present study, we used gnotobiotic rats associated with child microbiota, which represent an excellent model to study host-microbiota interactions in a well-controlled environment without confounding factors such as variations in diet or host genetic background ${ }^{(38-40)}$. A child was selected as the faecal donor due to the high prevalence of Fe deficiency and need for Fe supplementation in this age group ${ }^{(22,41)}$. The human microbiota-associated rats were fed diets differing in Fe concentration and source according to the standard Fe depletion-repletion study used to assess Fe bioavailability ${ }^{(42)}$. Changes in faecal microbiota composition were analysed during the study period, and caecal microbiota composition and metabolic activity, as well as gut inflammation, were assessed after killing the rats at the end of the study.

\section{Materials and methods}

\section{Rats and diets}

A total of forty germ-free female Fischer 344 rats were bred at the INRA facilities of Clermont-Ferrand-Theix, France. They were kept in sterile isolators with positive pressure over the entire trial period as described previously ${ }^{(40,43)}$. Rats were fed an irradiated standard diet for germ-free rodents (SAFE) and given free access to sterilised water. Rats were maintained pairwise in standard Macrolon cages under a $12 \mathrm{~h}$ light $-12 \mathrm{~h}$ dark cycle under constant temperature and humidity. At 5 weeks of age, rats were inoculated with a human faecal microbiota slurry obtained from a healthy volunteer (age 6 years) not treated with antibiotics 3 months before faecal sample collection. The faecal sample was processed within $6 \mathrm{~h}$ of defecation and maintained under anaerobic conditions. After 1000-fold dilution with an anaerobic mineral solution, $1 \mathrm{ml}$ of faecal slurry was orally inoculated into germfree rats. The microbiota was allowed to establish for 3 weeks while the rats were being fed a control diet with normal Fe concentrations equivalent to a standardised American Institute of Nutrition (AIN)-93G purified diet ${ }^{(44)}$ before starting the different feeding regimens.

All diets were produced by Dyets Inc. and based on a standard AIN-93G diet differing only in Fe concentration (Table S1, available online). The study set-up (Fig. 1) was designed according to the classical Fe depletion-repletion assay of Forbes et al. ${ }^{(42)}$ and comprised five different groups of rats. A 'control' group of rats $(n 8)$ was fed a regular AIN93G diet containing a mean of 37.6 (sem 0.9) $\mathrm{mg} \mathrm{Fe} / \mathrm{kg}$ diet from ferric citrate over the entire trial period of 16 weeks, while a 'Fe-deficient' group of rats $(n 8)$ was fed an Fe-deficient diet containing $2.9(\operatorname{sem} 0 \cdot 2) \mathrm{mg} \mathrm{Fe} / \mathrm{kg}$ diet. A further two groups of rats, ' 35 ppm Fe' group ( $n 8)$ and ' 70 ppm Fe' group ( $n$ 8), were first fed a Fe-deficient diet for 12 weeks and were then supplemented with either a $35 \mathrm{ppm}$ Fe diet containing $32 \cdot 2$ (SEM 1.0) $\mathrm{mg} \mathrm{Fe} / \mathrm{kg}$ diet from $\mathrm{FeSO}_{4}$ or a 70 ppm Fe diet containing 66.1 (sEM 7.8) $\mathrm{mg} \mathrm{Fe} / \mathrm{kg}$ diet from $\mathrm{FeSO}_{4}$ and ferric citrate to mimic Fe supplementation with two different Fe sources and concentrations for 4 weeks. A fifth group of rats, 'Fe-excess' group ( $n$ 8), was first fed the control diet and was then supplemented with the $70 \mathrm{ppm}$ Fe diet to mimic the impact of Fe supplementation on the gut microbiota not previously affected by a low-Fe diet and to investigate the effects of excess Fe on the gut microbiota. Once a week, body weight of each rat was measured and diet intake was assessed cage-wise (two rats). Fe concentration in the diets was assessed by atomic absorption spectrometry (SpectrAA-240K with GTA-120 Graphite Tube Atomizer, Varion Techtron) shortly before use. All procedures were carried out according to the European Directives on the protection of animals used for scientific purposes, 2010/63/ $\mathrm{EU}$, and the laboratory procedures were approved by the local ethics committee (CEMEAA 02). 


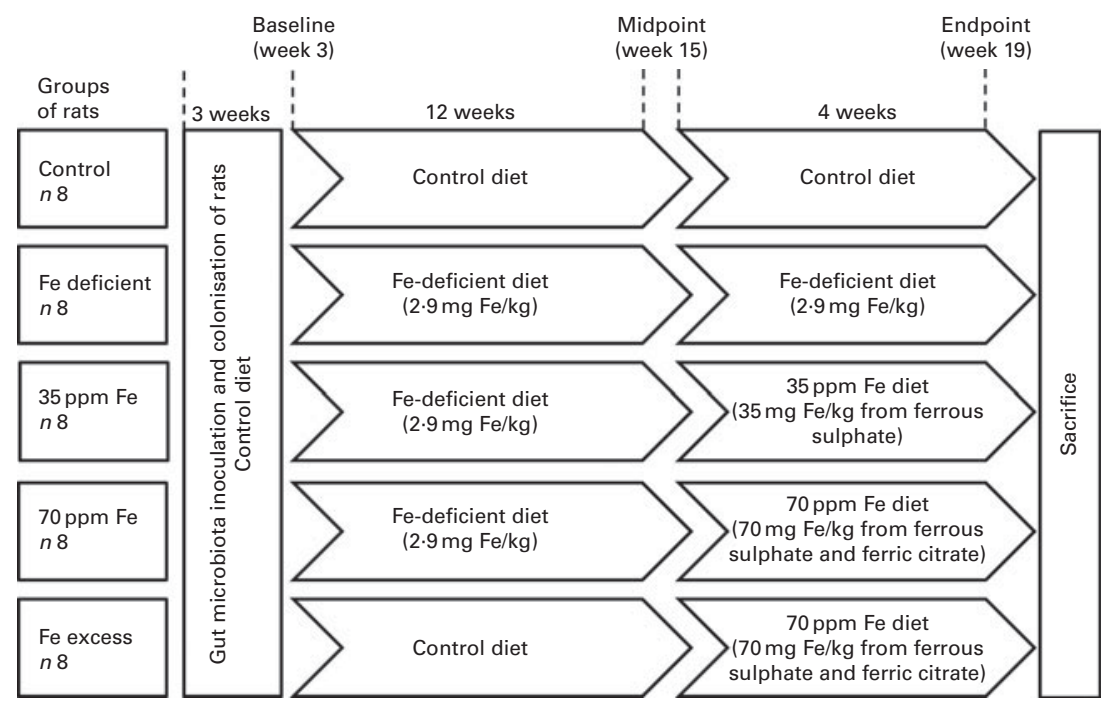

Fig. 1. Study set-up with different iron feeding regimens according to a classical iron depletion-repletion study design. Germ-free Fischer 344 rats $(n 40)$ were divided into five groups and inoculated with the same microbiota from a human volunteer. After 3 weeks of initial colonisation for gut microbiota establishment, diets differing only in iron concentration were fed to rats as outlined in the figure.

\section{Sample collection}

Faecal samples and blood samples of all rats were collected at baseline (week 3), after the first feeding period at midpoint (week 15) and at endpoint (week 19). Faecal samples were frozen at $-80^{\circ} \mathrm{C}$ until gut microbiota composition analysis by quantitative PCR (qPCR). Fe concentration in the faecal samples of the 35 ppm Fe-supplemented group, Fe-deficient group and control group at endpoint (week 19) was assessed by atomic absorption spectrometry (SpectrAA-240K with GTA120 Graphite Tube Atomizer, Varion Techtron). Blood samples were collected by the tail vein clip $\operatorname{method}^{(45)}$ and analysed immediately for Hb concentrations using a Hemocue 201 instrument (HemoCue). After killing the rats by $\mathrm{CO}_{2}$ inhalation, blood was collected again by cardiac puncture and centrifuged immediately, and serum was kept at $-80^{\circ} \mathrm{C}$ until ferritin measurements. Serum ferritin concentrations were assessed in duplicate using a rat ferritin ELISA kit according to the manufacturer's instructions (Immunology Consultants Laboratory, Inc.). Sections of the ileum, caecum and colon were removed, rinsed in PBS and immersed in $4 \%$ paraformaldehyde, and stored at $4^{\circ} \mathrm{C}$ until histological analyses. Caecal contents were also collected and frozen immediately at $-80^{\circ} \mathrm{C}$ for caecal microbiota metabolic activity and composition analyses.

\section{Histological analyses of ileal, caecal and colonic tissue samples}

All histological analyses were carried out by the Histology Analysis Platform of the Nantes Atlantic National College of Veterinary Medicine, Food Science and Engineering (Oniris, Nantes, France). After sectioning, ileal, caecal and colonic tissue samples were stained with haematoxylin-eosinsafranin and analysed microscopically using a Nikon Eclipse 5DI microscope connected to a Nikon DS-42-RI1 camera (200X or $400 \times$ magnification) (Nikon Instruments). Tissue samples were analysed for infiltration of immune cells, damage in crypt architecture, hyperaemia and mucosal erosion and were given a histological colitis score according to the severity of these parameters ${ }^{(46)}$. The veterinary pathologist examining the tissue samples was blinded to treatment.

\section{Faecal and caecal microbiota composition analysis using quantitative $P C R$}

Total genomic DNA from faecal samples and caecal content samples was extracted using the FastDNA SPIN Kit for Soil (MP Biomedicals). For the enumeration of total 16S rRNA gene copies and nine bacterial groups prevalent in the gut microbiota, namely Firmicutes, Bacteroides spp., Clostridium cluster IV, F. prausnitzii, E. hallii, Enterobacteriaceae, Lactobacillus/Leuconostoc/Pediococcus spp., Roseburia spp./ E. rectale, and sulphate-reducing bacteria (SRB), primers targeting the $16 \mathrm{~S}$ rRNA gene or a functional gene were used for qPCR carried out with an ABI PRISM 7500-PCR sequence detection system using $2 \times$ SYBR Green PCR Master Mix (Life Technologies), as described previously ${ }^{(27,47)}$. In short, PCR comprising $0 \cdot 2 \mu \mathrm{m}$ of each primer in $25 \mu \mathrm{l}$ volume were carried out in duplicate for each sample. In every run, a standard curve with serially diluted 16S rRNA gene or functional gene concentrations of a representative strain for each bacterial target group was included. SRB were enumerated with the same protocol using the primers dsrA_F336 (5'-CTG CGA ATA TGC CTG CTA CA-3') and dsrA_R533 (5'-TGG TCG ARC TTG ATG TCG TC- $\left.3^{\prime}\right)$ targeting the dissimilatory sulphite reductase subunit $\mathrm{A}^{(48)}$, and E. hallii $16 \mathrm{~S}$ rRNA gene copies were evaluated using the primers EhalF (5'-GCGTAGGTGGCAGTGCAA- $3^{\prime}$ ) and EhalR (5'-GCACCGRAGCCTATACGG-3') ${ }^{(49)}$.

\section{Pyrosequencing analysis}

Pyrosequencing analysis was carried out using caecal samples of the control group, Fe-deficient group and 
35 ppm Fe-supplemented group for three rat pairs in each group. Caecal content samples of the two rats housed in the same cage were pooled, and DNA was extracted using the FastDNA SPIN Kit for Soil (MP Biomedicals), resulting in three genomic DNA samples per analysed group of rats (total of nine genomic DNA samples). Pyrosequencing analysis was carried out by DNAVision (Charleroi, Belgium) using a 454 Life Sciences Genome Sequencer FLX instrument (Roche Applied Science), following previously described procedures $^{(27)}$. Resulting reads from the hypervariable gene region $\mathrm{V} 5-\mathrm{V} 6$ of the $16 \mathrm{~S}$ rRNA gene were assigned to samples according to their multiplex identifier tag, checked for the presence of primer sequences and fragment length $>200 \mathrm{bp}$. All reads not fulfilling these criteria were discarded, resulting in an average number of sequences of 5345 (SD 1929) per sample used in taxonomic assignment. Sequences were assigned on family and genus level using the Mothur ${ }^{(50)}$ and Greengenes $16 \mathrm{~S}$ reference database ${ }^{(51)}$. Moreover, sequences that did not align where $95 \%$ of the other sequences aligned were discarded. Chimera candidates were identified using the UCHIME implementation in Mothur ${ }^{(52)}$. Relative abundances of unassigned reads or reads assigned on family or genus level were calculated from the total number of reads matching the quality control criteria.

\section{Metabolite analysis}

Caecal content samples were centrifuged $(10000 \boldsymbol{g})$ and supernatants were diluted with MilliQ water before analysis by HPLC as described previously ${ }^{(53)}$. Fermentative metabolites (lactate, formate, acetate, propionate, butyrate, isovalerate, isobutyrate and valerate) and SCFA (acetate, propionate and butyrate) in each sample were analysed in duplicate.

\section{Statistical analysis}

Statistical analysis was carried out using JMP 8.0 and SPSS 18.0 (IBM SPSS, Inc.). All data were tested for normal distribution using the Shapiro-Wilk test and are expressed as means with their standard errors. Hb, ferritin, weight gain and gut microbiota concentrations for each metabolite and caecal qPCR data for each bacterial target were compared between the groups of rats using one-way ANOVA with a post boc Bonferroni test. Diet consumption was compared using nonparametric Kruskal-Wallis test. qPCR data on faecal microbiota composition were analysed over time for every bacterial target within each rat group using one-way repeated-measures ANOVA with Greenhouse-Geisser correction and post boc Bonferroni test. qPCR data were $\log _{10}$-transformed before statistical analysis. Histological colitis scores and pyrosequencing data were compared pairwise using the non-parametric Mann-Whitney $U$ test. $P$ values $<0.05$ were considered significantly different.

\section{Results \\ Iron status, faecal iron concentrations, weight gain and diet consumption}

Fe status of rats fed different Fe diets was assessed by blood $\mathrm{Hb}$ concentration measurements at midpoint (week 15) and at endpoint (week 19), while ferritin concentration was measured at endpoint (Table 1). Although rats were fed a highly Fe-deficient diet $(2.9 \mathrm{mg} \mathrm{Fe} / \mathrm{kg} \mathrm{diet})$ for up to 16 weeks after an initial colonisation period during which they were fed a standard diet, $\mathrm{Hb}$ and ferritin concentrations at endpoint (week 19) were not significantly different between the groups of rats. However, lighter caecal content colour and liver colour were observed in the majority of rats in the Fedeficient group (Fig. S1, available online). Weight gain was similar between all groups of rats during the first feeding period (weeks 3-15). During the second feeding period (weeks 15-19), rats in the control group exhibited the highest weight gain, which was significantly higher than that in rats in the $70 \mathrm{ppm}$ Fe-supplemented group and Fe-excess group possibly because the control group had the lowest weight at week 15 and therefore more growth potential during the second feeding period. Interestingly, diet consumption was similar between the groups of rats during the first and second feeding periods.

Table 1. $\mathrm{Hb}$ and ferritin concentrations, weight gain and dietary intake in rats fed a diet differing only in iron concentration at midpoint (week 15) and endpoint (week 19) ( $n 7-8$, each group)

(Mean values with their standard errors)

\begin{tabular}{|c|c|c|c|c|c|c|c|c|c|c|c|c|c|c|}
\hline \multirow[b]{2}{*}{ Groups } & \multicolumn{2}{|c|}{$\begin{array}{c}\mathrm{Hb} \text { week } 15^{\star} \\
(\mathrm{g} / \mathrm{l})\end{array}$} & \multicolumn{2}{|c|}{$\begin{array}{c}\mathrm{Hb} \text { week } 19^{*} \\
(\mathrm{~g} / \mathrm{l})\end{array}$} & \multicolumn{2}{|c|}{$\begin{array}{l}\text { Ferritin } \\
\text { week 19* } \\
(\mathrm{mg} / \mathrm{l})\end{array}$} & \multicolumn{2}{|c|}{$\begin{array}{l}\text { Weight gain } \\
\text { weeks } 3-15^{*} \\
\text { (g) }\end{array}$} & \multicolumn{2}{|c|}{$\begin{array}{l}\text { Weight gain } \\
\text { weeks } 15-19^{*} \\
\text { (g) }\end{array}$} & \multicolumn{2}{|c|}{$\begin{array}{l}\text { Diet consumed } \\
\text { weeks } 3-15 \dagger \\
\text { (g) }\end{array}$} & \multicolumn{2}{|c|}{$\begin{array}{l}\text { Diet consumed } \\
\text { weeks } 15-19 \dagger \\
\text { (g) }\end{array}$} \\
\hline & Mean & SEM & Mean & SEM & Mean & SEM & Mean & SEM & Mean & SEM & Mean & SEM & Mean & SEM \\
\hline Control & $168 \cdot 9^{\mathrm{a}}$ & 4.5 & 153.4 & 4.4 & 3.3 & 0.6 & 38.4 & 2.5 & $15 \cdot 1^{\mathrm{a}}$ & 1.8 & $716 \cdot 1$ & 11.9 & $262 \cdot 1$ & $5 \cdot 0$ \\
\hline Deficient & $154 \cdot 8^{a, b}$ & 4.5 & 147.9 & 2.5 & 1.9 & 0.8 & $37 \cdot 1$ & $2 \cdot 2$ & $9 \cdot 7^{\mathrm{a}, \mathrm{b}}$ & 1.6 & 751.3 & $16 \cdot 1$ & 254.2 & $6 \cdot 4$ \\
\hline $35 \mathrm{ppm} \mathrm{Fe}$ & $150 \cdot 9^{b}$ & 2.5 & 148.6 & 3.3 & 2.5 & 0.6 & $40 \cdot 0$ & 2.6 & $10 \cdot 2^{a, b}$ & 1.3 & 747.9 & $12 \cdot 5$ & 273.6 & 3.9 \\
\hline $70 \mathrm{ppm} \mathrm{Fe}$ & $151 \cdot 6^{\mathrm{b}}$ & $3 \cdot 1$ & $146 \cdot 9$ & $2 \cdot 5$ & $1 \cdot 7$ & 0.4 & $36 \cdot 6$ & $4 \cdot 2$ & $8 \cdot 7^{b}$ & 0.9 & $770 \cdot 2$ & $24 \cdot 2$ & 255.3 & $8 \cdot 7$ \\
\hline $\mathrm{Fe}$ excess & $150 \cdot 7^{b}$ & $3 \cdot 8$ & $147 \cdot 1$ & 1.6 & 4.4 & 1.4 & 41.0 & 1.3 & $8.9^{b}$ & 1.0 & $747 \cdot 7$ & $13 \cdot 1$ & 254.6 & 5.0 \\
\hline$P$ & 0.007 & & 0.549 & & 0.073 & & 0.761 & & 0.010 & & 0.380 & & 0.535 & \\
\hline
\end{tabular}

a,b Mean values within a column with unlike superscript letters were significantly different $(P<0.05)$

* Differences among groups were tested by ANOVA and post hoc Bonferroni test.

†Differences among groups were tested by non-parametric Kruskal-Wallis test. 

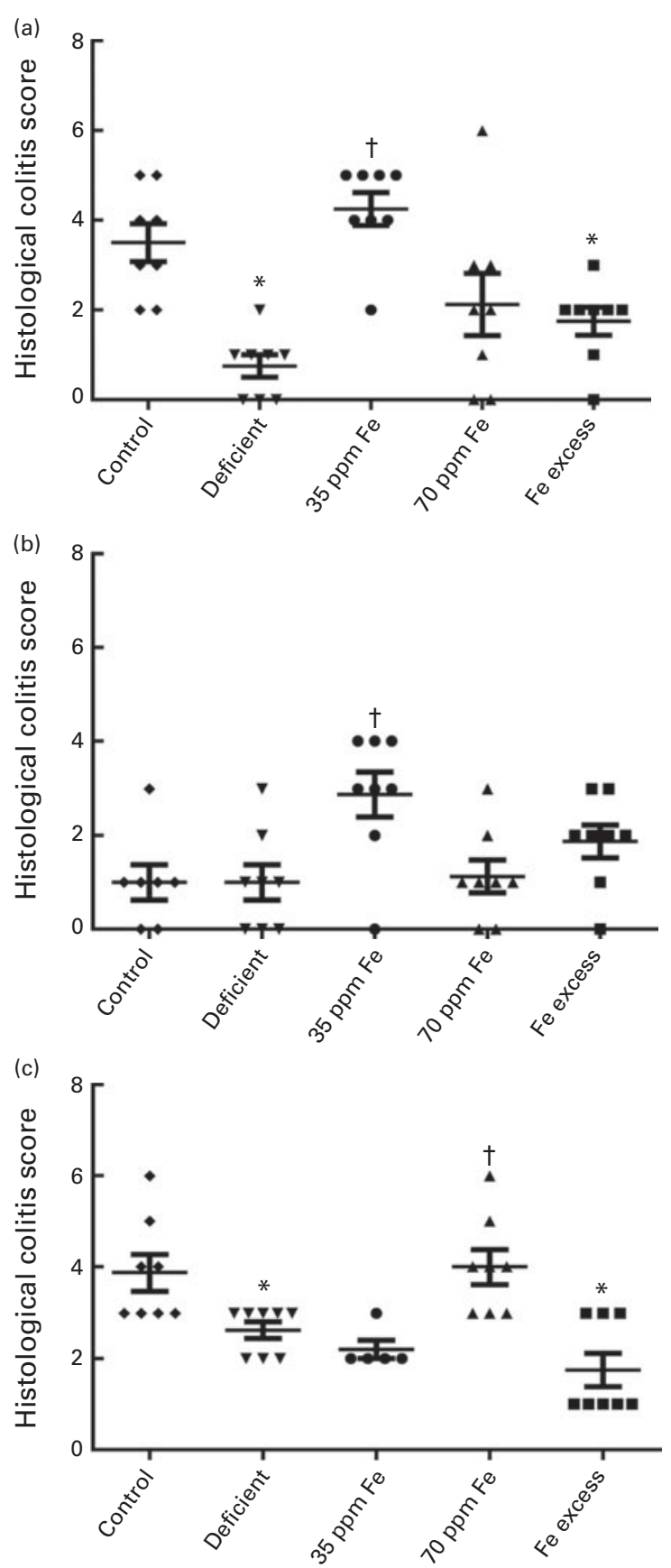

Fig. 2. Histological colitis scores of (a) ileum, (b) caecum and (c) colon of rats fed diets differing in iron concentration. Colitis scores were obtained by light microscopy after haematoxylin/eosin/safranin staining and investigation of infiltration of immune cells, damage in crypt architecture, hyperaemia and mucosal erosions. Each dot represents one rat. Horizontal bars are means, with their standard errors. * Mean value was significantly different from that of the control group $(P<0.05$; non-parametric Mann-Whitney $U$ test). $\dagger$ Mean value was significantly different from that of the iron-deficient group $(P<0.05$; non-parametric Mann-Whitney $U$ test).

At endpoint, Fe concentration in the faecal samples of rats in the Fe-deficient group (20 (SEM 3) $\mathrm{mg} \mathrm{Fe} / \mathrm{kg}$ ) was significantly lower than that in the faecal samples of rats in the $35 \mathrm{ppm}$ Fe-supplemented group (185 (sEm 14) $\mathrm{mg} \mathrm{Fe} / \mathrm{kg}$, $P<0 \cdot 001$ ) and control group (184 (sem 7) mg Fe/kg, $P<0 \cdot 001$ ).

\section{Effect of different iron concentrations in the diet on histological colitis scores}

Ileum, caecum and colon of rats were collected after killing them, preserved in paraformaldehyde, and investigated by light microscopy for infiltration of immune cells, damage in crypt architecture, hyperaemia and mucosal erosion to evaluate histological colitis scores (Fig. 2(a)-(c)). In general, no severe colitis was detected in the ileum, caecum or colon of all rat groups, and the highest average score was 4.3 (sEm 0.4) (ileum, 35 ppm Fe-supplemented group) of a maximum possible score of 11 , indicating very light colitis. Rats fed an Fe-deficient diet had the lowest ileum histological colitis scores (score of $0 \cdot 8$ (SEM $0 \cdot 3)$ ), which were significantly lower than those of the control rats $(3.5$ (SEM $0 \cdot 4), P=0 \cdot 001)$ and $35 \mathrm{ppm}$ Fe-supplemented rats (4.3 (SEM 0.4), $P=0.0009)$ (Fig. 2(a)). Caecum histological colitis scores were similar between all groups of rats and rats in the $35 \mathrm{ppm} \mathrm{Fe-}$ supplemented group had the highest scores (2.9 (SEM 0.5)) (Fig. 2(b)). Supplementation with $35 \mathrm{ppm}$ Fe after feeding an Fe-deficient diet (35 ppm Fe-supplemented group) significantly increased the caecum histological colitis scores compared with those of rats in the Fe-deficient group. Surprisingly, when a diet with 70 ppm Fe was fed after feeding an Fe-deficient diet ( $70 \mathrm{ppm}$ Fe-supplemented group), no increase in histological colitis scores was observed. The opposite observation was made in the colon, wherein the 70 ppm Fe-supplemented group had higher histological colitis scores (4.0 (SEM 0.4)) than the Fe-deficient group (Fig. 2(c)). Moreover, differences in histological colitis scores between the different parts of the intestine (ileum-caecum-colon) were observed with a lower histological colitis score being observed for the caecum in the control and $70 \mathrm{ppm} \mathrm{Fe}$ supplemented groups and an increase in histological colitis scores from the ileum to the colon being observed in the Fe-deficient group. By contrast, a significant decrease in histological colitis scores from the ileum to the colon was observed in rats in the $35 \mathrm{ppm}$ Fe-supplemented group, while no changes were observed in those in the $\mathrm{Fe}$ excess group.

\section{Caecal and faecal microbiota composition in rats fed diets differing in iron concentration}

Caecal contents of all rats were collected after killing them and the extracted DNA was investigated by qPCR to enumerate different bacterial groups present in the gut microbiota (Fig. 3) and by 454-pyrosequencing (Fig. 4(a)-(c)) to assess gut microbiota diversity and composition. qPCR did not reveal changes in the log number of total 16S rRNA gene copies or Firmicutes 16S rRNA gene copies/g caecum between the different groups of rats fed different Fe diets. The bacterial community in all rats was dominated by Firmicutes, including Clostridium cluster IV, followed by Bacteroides spp. (Fig. 3). This finding was confirmed by 454-pyrosequencing in rats in the control group, Fe-deficient group and $35 \mathrm{ppm}$ Fe-supplemented group, where the phylum Firmicutes (54.5-82.6\%, data not shown) dominated, followed by 


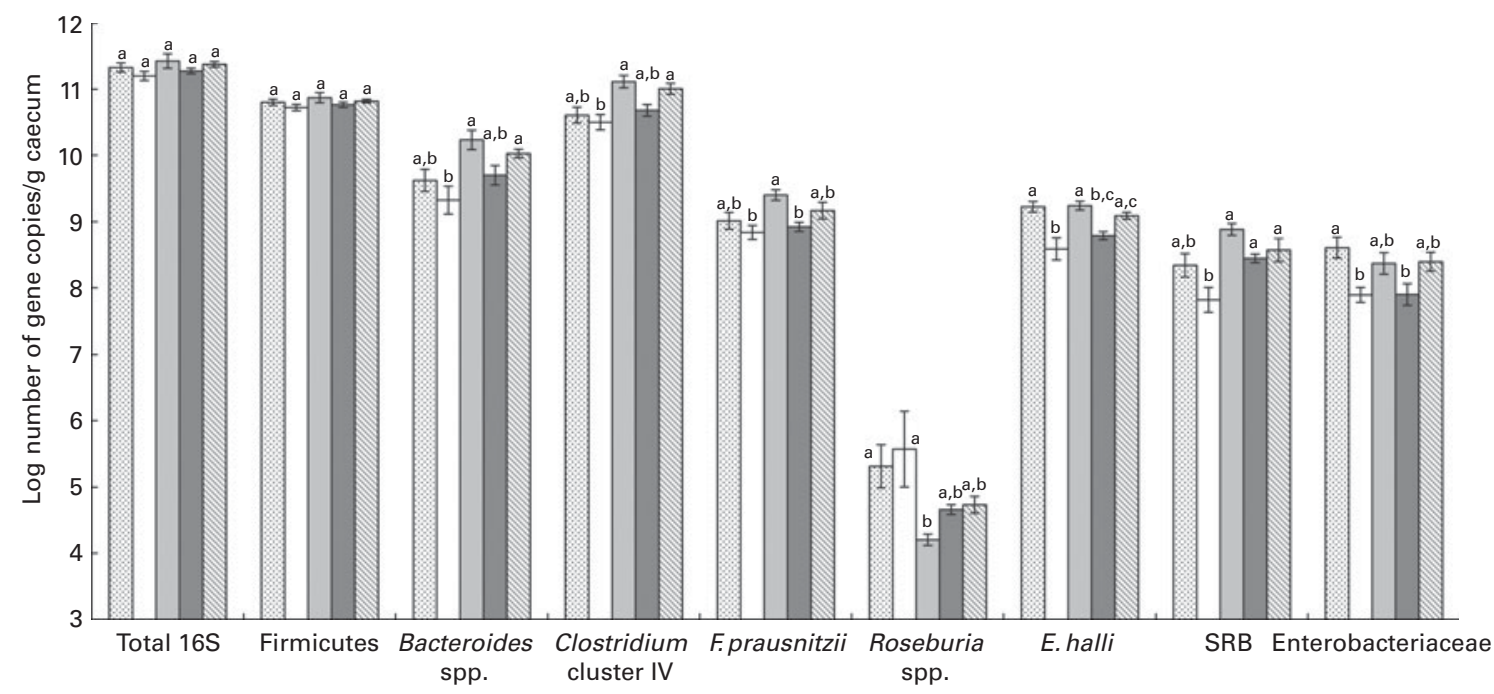

Fig. 3. Caecal microbiota composition after killing in rats fed diets differing only in iron concentration. Bacterial groups were enumerated with specific bacterial primers targeting the 16S rRNA gene or a specific functional gene by quantitative PCR. Values are means ( $n 8$; iron excess, $n 7)$, with their standard errors represented by vertical bars. ${ }^{\mathrm{a}, \mathrm{b}, \mathrm{c}}$ Mean values within the same bacterial group with unlike letters were significantly different $(P<0.05$; ANOVA followed by post hoc

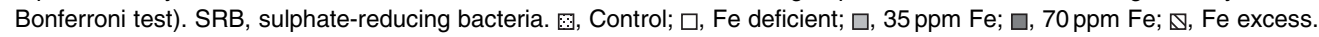

Bacteroidetes $(14 \cdot 3-42 \cdot 0 \%$, data not shown). On the taxonomic family level, these rats had the highest relative abundance $(34 \cdot 1-43 \cdot 8 \%)$ of Lachnospiraceae (Firmicutes) (Fig. 4(a)).

The levels of dominant bacterial groups (Firmicutes, Bacteroides spp. and Clostridium cluster IV) in caecum were similar in rats in the control and Fe-deficient groups. However, the levels of $E$. hallii and Enterobacteriaceae were significantly lower in rats fed an Fe-deficient diet (8.59 (SEM 0.17) and 7.89 (SEM $0 \cdot 11$ ), respectively; mean log 16S rRNA gene copies/g caecum) than in the control rats (9.22 (SEM 0.08) and 8.61 (sEm 0-16), respectively) (Fig. 3). Moreover, a significantly lower abundance of Bilophila spp. (0.36 (SEM 0.11)\%) and Coprococcus spp. (0.74 (SEM 0.07)\%) was observed in rats in the Fe-deficient group than in those in the control group (Bilophila spp., 0.87 (SEM 0.19)\%; Coprococcus spp. 2.11 (SEM 0.58)\%) by 454-pyrosequencing (Fig. 4(c)). Bacteroides spp., Clostridium cluster IV, F. prausnitzii, E. hallii and SRB were significantly increased in the caecum of rats in the $35 \mathrm{ppm} \mathrm{Fe}-$ supplemented group than in that of rats in the Fe-deficient group; however, bacterial concentrations did not increase to higher levels compared with those in the control group (Fig. 3). The 454-pyrosequencing analysis revealed a significantly higher relative abundance of Lachnospiraceae $(43.81$ (SEm 2.79)\%, Fe-deficient group: $34 \cdot 14$ (SEM 1.97)\%), Ruminococcaceae $(12 \cdot 15$ (SEM 1.30)\%, Fe-deficient group: $8 \cdot 25$ (SEM $0.38) \%$ ) and Veillonellaceae (0.46 (sEm 0.05)\%, Fe-deficient group: 0.29 (SEM 0.02$) \%$ in the $35 \mathrm{ppm}$ Fe-supplemented group than in the Fe-deficient group (Fig. 4(a)). On the genus level, the abundance of Coprococcus spp. (2.21 (sEm 0.43)\%, Fe-deficient group: 0.74 (SEm 0.07)\%) and Bilophila spp. (0.77 (SEM 0.05)\%, Fe-deficient group: 0.36 (SEM 0.11)\%) was significantly increased, while that of Enterococcus spp. (0.05 (SEM 0.03)\%, Fe-deficient group: 1.24 (SEM 1.03)\%) and Turicibacter spp. (0.23 (sEM 0.14)\%, Fe-deficient group:
1.41 (SEM 0.38)\%) was significantly decreased in the $35 \mathrm{ppm}$ Fe-supplemented group than in the Fe-deficient group (Fig. 4(b) and (c)). Bacterial populations in rats in the $70 \mathrm{ppm}$ Fe-supplemented group and the Fe-excess group did not differ when compared with those in rats in the Fe-deficient group or control group, respectively (Fig. 3).

Faecal samples collected at baseline (week 3), midpoint (week 15) and endpoint (week 19) shortly before killing were analysed for microbial composition using qPCR to enumerate prevalent bacterial groups (Table S2, available online). During the entire trial period of 16 weeks, the faecal microbiota was very stable, with changes in the log numbers of $16 \mathrm{~S}$ rRNA gene copies of dominant bacterial groups per $\mathrm{g}$ faeces being less than $1 \mathrm{log}$, and no direct correlations between dietary $\mathrm{Fe}$ concentrations and faecal microbiota composition could be extracted.

\section{Effect of different iron diets on caecal microbiota metabolic activity}

Fermentative metabolites and SCFA concentrations measured by HPLC (Fig. 5) were not different between rats in the Fe-deficient group and those in the control group. However, supplementation with $\mathrm{Fe}$ at the 35 and $70 \mathrm{ppm}$ levels affected the caecal microbiota metabolic profile profoundly. Fermentative metabolites and acetate and propionate concentrations were all significantly increased in rats in the $35 \mathrm{ppm}$ Fe-supplemented group (83.2 (SEM 10.1), 33.7 (SEM 2.3) and 15.3 (sem 0.9) $\mathrm{mm}$, respectively) and the $70 \mathrm{ppm}$ Fesupplemented group (62.0 (SЕм 2.6), 33.1 (SЕм 1.6) and 14.5 (SEM 0.9) $\mathrm{mm}$, respectively) than in those in the Fe-deficient group (39.6 (SEM 1.6), 23.7 (SEM 1.2) and 9.5 (SEm 0.5) mm, respectively). Moreover, butyrate concentration was significantly increased in the $35 \mathrm{ppm}$ Fe-supplemented group (29.5 (SEM 7.5) mM) compared with 
(a)

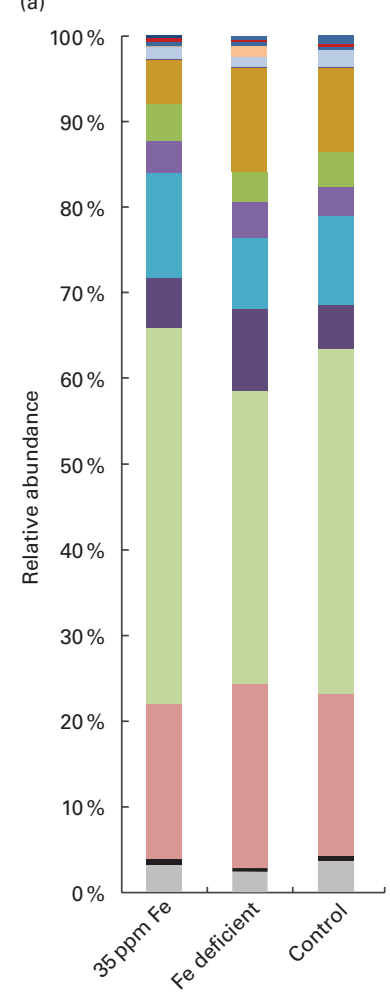

(b)

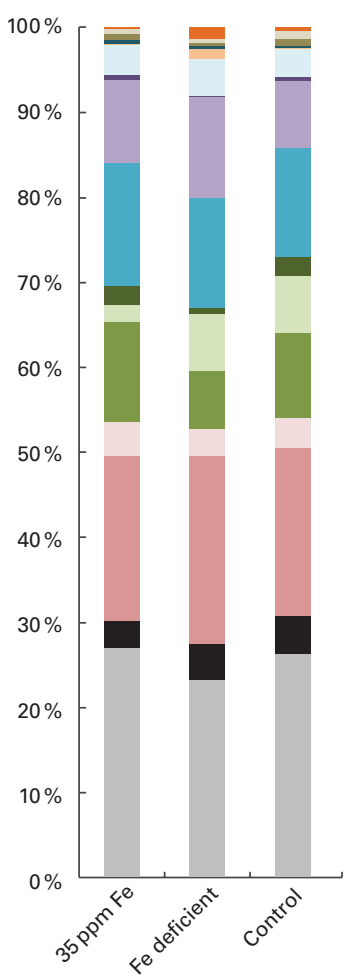

(c)

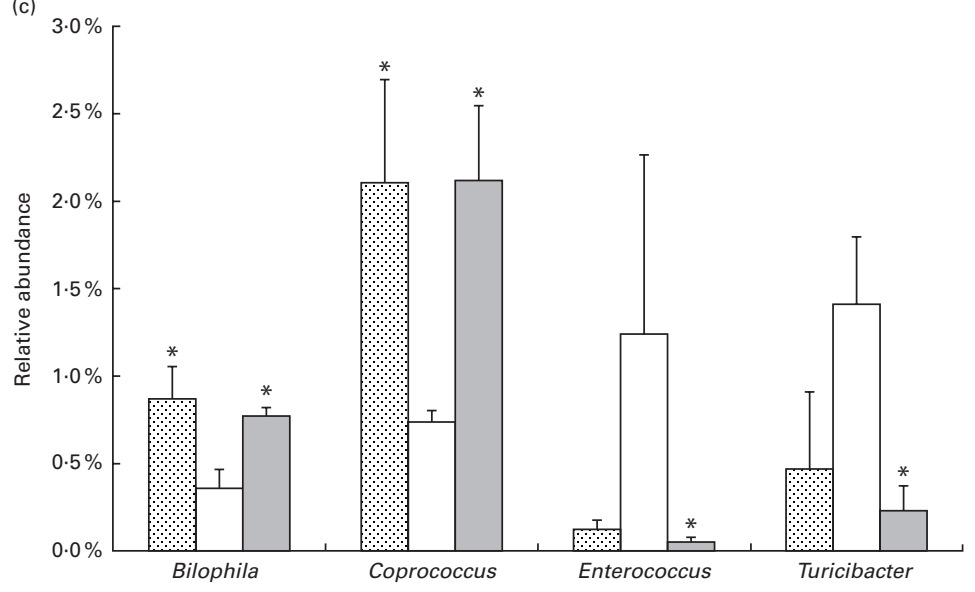

Fig. 4. Caecal microbiota composition of rats fed the $35 \mathrm{ppm}$ iron diet ( $n 3$ samples), rats fed an iron-deficient diet ( $n 3$ samples) and rats fed the control diet ( $n 3$ samples). Relative abundances of (a) bacterial families and (b) genera identified by 454-pyrosequencing analysis. (c) Relative abundance of subdominant genera Bilophila, Coprococcus, Enterococcus and Turicibacter. Values are means, with their standard errors represented by vertical bars. * Mean values were significantly different from those of the iron-deficient group $(P<0.05$; non-parametric Mann-Whitney $U$ test). (a) $\square$, Verrucomicrobiaceae; $\square$, Veillonellaceae;

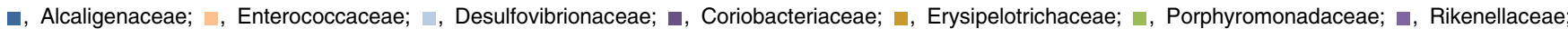

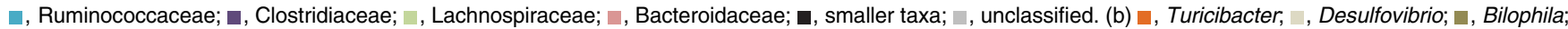

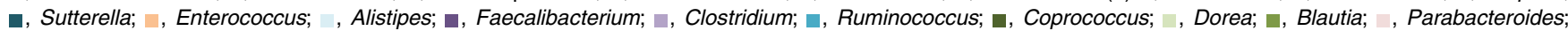

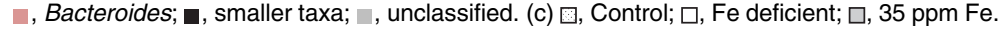

the Fe-deficient group ( $4.8($ SEm $0 \cdot 8) \mathrm{mm})$. Propionate and butyrate concentrations in the $35 \mathrm{ppm}$ Fe-supplemented group were even higher than those in the control group. On the other hand, supplementation with 70 ppm Fe led to no significant increase in butyrate concentration compared with that in rats in the Fe-deficient group. Propionate (15.4 (SEM $0.7) \mathrm{mm}$ ) and butyrate (16.7 (SEM 3.9) $\mathrm{mm}$ ) concentrations were significantly increased in the Fe-excess group compared with the control group (11.7 (SEM 0.8) and 3.3 (SEM 0.6) mM, respectively).

\section{Discussion}

The results of the present study carried out using human gut microbiota-associated rats provide a new insight into the highly complex interactions between the host, the gut microbiota and Fe. Colonisation of rats with human gut microbiota was successful, and dominant bacterial groups were present at similar levels in rats at baseline and in the donor microbiota as described previously ${ }^{(36,40)}$. The use of one faecal donor also led to highly similar microbiotas in all rats, whereas in humans variability in microbiota composition between individuals is high and may potentially mask smaller effects ${ }^{(54)}$. Feeding regimens were set up according to the classical $\mathrm{Fe}$ depletion-repletion study ${ }^{(42)}$ in which Fe depletion is started in very young rats (usually 3 weeks old), which have small body Fe stores and high Fe needs for growth, leading to severe Fe-deficiency anaemia ${ }^{(28,42)}$.

Surprisingly, in the present study, the 12- and 16-week depletion periods were insufficient to induce Fe-deficiency anaemia. This may be because the rats were 8 weeks of age when the intervention was started (instead of the usual 3 weeks) and had time to build up adequate Fe stores. Drawing on these stores allowed them to maintain $\mathrm{Hb}$ concentrations during the depletion period. However, rats in the Fe-deficient group had caecal contents of a lighter colour, suggesting a mild degree of $\mathrm{Fe}$ depletion (Fig. S1, available online). In addition, Fischer 344 rats are known to be more resistant to Fe deficiency than the Sprague-Dawley rats usually used in a $\mathrm{Hb}$ depletion-repletion study ${ }^{(55)}$. At endpoint, faecal Fe concentrations in rats were higher than dietary Fe concentrations, which may be explained by a concentration effect of Fe in the gut lumen during water and nutrient absorption and a possible Fe loss in the host by sloughed enterocytes.

Dietary Fe supplementation has been shown to be associated with increased inflammatory reactions of the gut mucosa possibly generated by the production of reactive oxygen species in the presence of elevated luminal $\mathrm{Fe}$ 


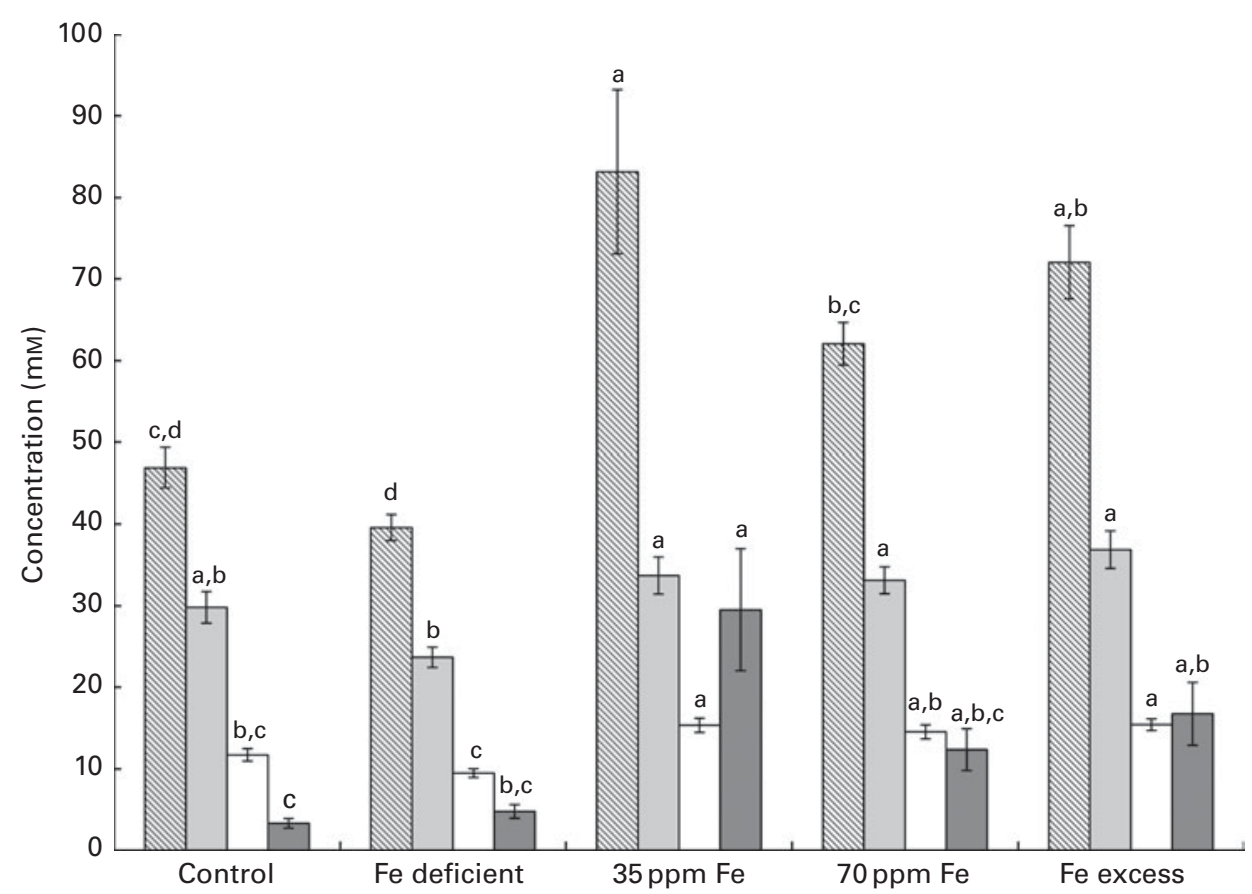

Fig. 5. Caecal fermentative metabolites and acetate, propionate and butyrate concentrations after killing in different groups of rats. Concentrations were measured by HPLC in caecum water samples in duplicate. Values are means ( $n$; 35 ppm iron, $n 7$ ), with their standard errors represented by vertical bars. a,b,c,d Mean values of the same metabolite with unlike letters were significantly different $(P<0.05$; one-way ANOVA followed by post hoc Bonferroni test). $\nabla$, Fermentative metabolites; $\square$, acetate; $\square$, propionate; $\square$, butyrate.

concentration, which has been observed in inflammatory bowel disease patients and rat models ${ }^{(56-58)}$, while other studies suggest an involvement of gut bacterial composition changes due to Fe concentrations ${ }^{(25,59)}$. Werner et al. ${ }^{(25)}$ found that a low-Fe diet completely inhibited gut inflammation in a mouse colitis model, while Fe supplementation in wild-type mice had no effect on colitis scores. In agreement, the present study showed lower histological colitis scores of ileum and colon in rats fed an Fe-deficient diet while Fe supplementation with $35 \mathrm{ppm}$ Fe but not with 70 ppm Fe caused light colitis in ileum and caecum. Therefore, no clear inflammation pattern related to Fe concentrations could be detected in the investigated rats. Fe supplementation in school children in Côte d'Ivoire has been found to clearly increase calprotectin concentrations, but in an environment with a high risk of pathogen exposure ${ }^{(30)}$. We speculate that Fe supplementation in the range of physiological levels may not lead to mucosal inflammation unless other influencing factors are present such as a high contamination with pathogens or an inflammatory pre-set of the host.

Faecal microbiota composition in all rats was very stable over time. Because rats had normal Fe status, we speculated that especially under an Fe-deficient diet regimen, bacteria in the colon could sequester Fe from sloughed enterocytes. Therefore, caecal contents were analysed, in which Fe might have a more direct impact on the microbiota. There was no difference in gut microbiota metabolic activity in caecum between rats in the Fe-deficient group and those in the control group. However, we found that low luminal Fe concentrations slightly affected the gut microbiota composition and decreased the relative abundances of Bilophila spp., E. hallii and Coprococcus spp. most probably due to Fe-dependent enzymes such as hydrogenases in their metabolic pathways $^{(9,60,61)}$.

The findings of the present study are in contrast to previous findings in highly Fe-deficient rats and also to the results of an in vitro colonic fermentation experiment ${ }^{(24,27)}$. In both experiments, very-low-Fe conditions had caused major dysbiosis of the gut microbiota and especially decreased butyrate and propionate production. Moreover, it has been shown that changes in host Fe metabolism in mice and humans have effects on the gut microbiota without changing dietary Fe concentrations ${ }^{(32,62)}$. However, in the present study, hosts were not Fe deficient and it is possible that host Fe plays an important role in the maintenance of the composition and metabolic function of the gut microbiota as part of the symbiosis between the microbiota and the host. Indeed, during low-Fe diet feeding, faecal $\mathrm{Fe}$ concentrations were $20 \mathrm{mg} \mathrm{Fe} / \mathrm{kg}$ faeces, which could be provided by unabsorbed dietary Fe and by sloughed enterocytes from the host. These faecal Fe concentrations are probably adequate to maintain the gut microbiota as has been shown in our previous in vitro studies $^{(27)}$.

Fe supplementation with $35 \mathrm{mg} \mathrm{Fe} / \mathrm{kg}$ diet from $\mathrm{FeSO}_{4}$ (35 ppm Fe-supplemented group) had a strong effect on both the caecal microbiota composition and metabolic activity, especially increasing the abundance of Bacteroides spp. and butyrate producers such as Clostridium cluster IV members, e.g. F. prausnitzii, and Coprococcus spp. Moreover, a decrease in the relative abundances of Turicibacter spp. and 
Enterococcus spp., both of which are opportunistic pathogens $^{(63,64)}$, was observed during feeding of the $35 \mathrm{ppm} \mathrm{Fe}$ diet than during feeding of a Fe-deficient diet. Enterococci most probably had a growth advantage under low-Fe conditions due to their restricted need for $\mathrm{Fe}$ and lost this advantage during Fe supplementation. Similar observations were made in mice in which feeding a diet containing $\mathrm{Fe}$ led to an increase in the abundance of Bacteroides spp. and to a decrease in that of Turicibacter spp. compared with feeding a Fe-free diet ${ }^{(25)}$.

The concentrations of acetate, propionate and especially butyrate were significantly increased due to Fe supplementation compared with those in rats in the Fe-deficient group. In a previous study, Fe supplementation has been found to lead to a significant increase in butyrate and propionate concentrations in highly Fe-deficient rats ${ }^{(24)}$. Moreover, Fe-deficient conditions in the same rat study and also in a colonic in vitro fermentation study led to a decrease in butyrate and propionate production ${ }^{(27)}$. These observations suggest that Fe is a crucial element for butyrate and propionate production in strict anaerobic gut bacteria. Indeed, in the butyrate production pathway, oxidoreductases and hydrogenases are involved ${ }^{(8,14,65)}$, which are often Fe dependent, and under conditions of optimal bioavailable Fe concentrations, the conversion of dietary or host carbons into end metabolites such as butyrate by gut bacteria may be enhanced. Studies in bioreactors with mixed strict-anaerobe cultures have shown that increasing Fe concentrations lead to a higher $\mathrm{H}_{2}$ and butyrate yield per carbon source ${ }^{(29,66,67)}$. Indeed, when calculating the total carbon output from bacterial fermentation in the form of SCFA, the diet with $35 \mathrm{mg} \mathrm{Fe} / \mathrm{kg}$ diet from $\mathrm{FeSO}_{4}$ (35 ppm Fe-supplemented group) led to more than double the carbon output than the control diet or a Fe-deficient diet, mainly due to the promotion of butyrate production, although diet consumption was similar in these groups of rats. SCFA can provide up to an additional $10 \%$ of daily dietary energy to the host from indigestible compounds, such as fibres ${ }^{(68)}$. This promotion of carbon output due to Fe supplementation could increase the energy source of plant-based diets, which are mainly consumed in developing countries, and therefore might contribute to weight gain in malnourished individuals.

A significant increase in caecal butyrate and propionate concentrations along with a decrease in the abundance of possible opportunistic pathogens due to $35 \mathrm{ppm}$ Fe supplementation could lead to beneficial effects on host gut health. Propionate produced by several Bacteroides spp. has been shown to be involved in the regulation of satiety ${ }^{(69)}$. Butyrate is the main energy source for colonocytes, and it can inhibit NF-кB activation and therefore decrease inflammatory responses ${ }^{(70,71)}$. Moreover, butyrate has anti-carcinogenic effects through the promotion of apoptosis and inhibition of proliferation ${ }^{(72)}$. Interestingly, no correlation between increased butyrate concentrations and decreased colitis scores was observed in the present study, but the anti-inflammatory effects of butyrate might be much more visible in mucosa with an inflammatory pre-set. Moreover, the abundance of $\mathrm{H}_{2}$-utilising and potentially toxic $\mathrm{H}_{2} \mathrm{~S}$-producing $\mathrm{SRB}$ was increased with Fe supplementation in the present study possibly due to the promotion of the production of $\mathrm{H}_{2}$, a by-product of the butyrate production pathway ${ }^{(65)}$. SRB have been identified as possible contributors of different digestive pathologies such as inflammatory bowel syndrome and inflammatory bowel disease ${ }^{(40,73-76)}$, and $\mathrm{H}_{2} \mathrm{~S}$ has been shown to affect metabolic functions in colonocytes and to cause DNA damage ${ }^{(74,76)}$. However, other studies have suggested a protective role of $\mathrm{H}_{2} \mathrm{~S}$ in the epithelial layer during inflammation and identified $\mathrm{H}_{2} \mathrm{~S}$ as an important mediator for intracellular processes ${ }^{(77,78)}$. Therefore, it remains difficult to directly associate an increase in SRB counts and $\mathrm{H}_{2} \mathrm{~S}$ production with negative effects on the gut mucosa, and no negative modulation of the gut mucosa could be detected in the present study.

In conclusion, the present study carried out using human gut microbiota-associated rats investigated the effects of dietary Fe concentrations on the gut microbiota. Our data suggest that an Fe-deficient diet alone may have no major effects on dominant bacterial populations or gut microbiota metabolic activity in a host, which is not Fe deficient based on blood parameters as observed in the study. By contrast, Fe supplementation with $35 \mathrm{mg} \mathrm{Fe} / \mathrm{kg}$ diet from $\mathrm{FeSO}_{4}$ promoted dominant bacterial groups and slightly increased SRB, while the abundance of potential opportunistic pathogens was decreased. Moreover, Fe supplementation strongly increased the metabolic activity of the gut microbiota. Histological colitis scores remained very low despite Fe supplementation, indicating that $\mathrm{Fe}$ alone does not lead to gut inflammation. Thus, we suggest that Fe supplementation might confer additional health benefits on the host by stimulating the gut microbiota without an inflammatory gut mucosa and in a relatively pathogen-free environment.

\section{Supplementary material}

To view supplementary material for this article, please visit http://dx.doi.org/10.1017/S000711451400021X

\section{Acknowledgements}

The authors thank Christophe De Martrin and Gérard Vert for their assistance in animal care and maintenance as well as Rainer Follador (Microsynth AG) for bioinformatics analysis of the pyrosequencing data.

The present study was funded by grants from the Swiss National Science Foundation (project number: 310030_ 127272, Bern, Switzerland) and the Eunice Kennedy Shriver National Institute of Child Health and Human Development (award number: U01HD0 64921). The Swiss National Science Foundation and the Eunice Kennedy Shriver National Institute of Child Health and Human Development had no role in the design and analysis of the study or in the writing of this article.

The authors' contributions are as follows: A. D., C. C., A. B.-D., C. D. and C. L. designed the study; A. D., C. C., C. D. and V. T. P. carried out the study; A. D. and V. T. P. analysed the data; A. D. wrote the manuscript; C. C., A. B.-D., C. D., M. B. Z. and C. L. edited the manuscript.

None of the authors has any conflicts of interest to declare.

tation in the present study possibly due to the promotion of 


\section{References}

1. Guarner F (2006) Enteric flora in health and disease. Digestion 73, Suppl. 1, 5-12.

2. Sekirov I, Russell SL, Antunes LC, et al. (2010) Gut microbiota in health and disease. Physiol Rev 90, 859-904.

3. Kau AL, Ahern PP, Griffin NW, et al. (2011) Human nutrition, the gut microbiome and the immune system. Nature $\mathbf{4 7 4}$ 327-336.

4. Stecher B \& Hardt WD (2011) Mechanisms controlling pathogen colonization of the gut. Curr Opin Microbiol 14, 82-91.

5. Louis P, McCrae SI, Charrier C, et al. (2007) Organization of butyrate synthetic genes in human colonic bacteria: phylogenetic conservation and horizontal gene transfer. FEMS Microbiol Lett 269, 240-247.

6. Hoyles L \& Wallace RJ (2010) Gastrointestinal tract: intestinal fatty acid metabolism and implications for health. In Handbook of Hydrocarbon and Lipid Microbiology, pp. 3119-3132 [K Timmis, editor]. Berlin: Springer.

7. Scott KP, Gratz SW, Sheridan PO, et al. (2013) The influence of diet on the gut microbiota. Pharmacol Res 69, 52-60.

8. Louis P \& Flint HJ (2009) Diversity, metabolism and microbial ecology of butyrate-producing bacteria from the human large intestine. FEMS Microbiol Lett 294, 1-8.

9. Pryde SE, Duncan SH, Hold GL, et al. (2002) The microbiology of butyrate formation in the human colon. FEMS Microbiol Lett 217, 133-139.

10. Roediger WE (1980) Role of anaerobic bacteria in the metabolic welfare of the colonic mucosa in man. Gut 21, 793-798.

11. Hamer HM, Jonkers D, Venema K, et al. (2008) Review Article: the role of butyrate on colonic function. Aliment Pharmacol Ther 27, 104-119.

12. Gantois I, Ducatelle R, Pasmans F, et al. (2006) Butyrate specifically down-regulates salmonella pathogenicity island 1 gene expression. Appl Environ Microbiol 72, 946-949.

13. Flint HJ, Duncan SH, Scott KP, et al. (2007) Interactions and competition within the microbial community of the human colon: links between diet and health. Environ Microbiol 9, 1101-1111.

14. De Vuyst L \& Leroy F (2011) Cross-feeding between bifidobacteria and butyrate-producing colon bacteria explains bifidobacterial competitiveness, butyrate production, and gas production. Int J Food Microbiol 149, 73-80.

15. Gupta SS, Mohammed MH, Ghosh TS, et al. (2011) Metagenome of the gut of a malnourished child. Gut Pathog 3, 7.

16. Smith MI, Yatsunenko T, Manary MJ, et al. (2013) Gut microbiomes of Malawian twin pairs discordant for kwashiorkor. Science 339, 548-554.

17. Lin A, Bik EM, Costello EK, et al. (2013) Distinct distal gut microbiome diversity and composition in healthy children from Bangladesh and the United States. PLOS ONE 8, e 53838.

18. Yatsunenko T, Rey FE, Manary MJ, et al. (2012) Human gut microbiome viewed across age and geography. Nature 486, 222-227.

19. Grzeskowiak L, Collado MC, Mangani C, et al. (2012) Distinct gut microbiota in southeastern African and northern European infants. J Pediatr Gastroenterol Nutr 54, 812-816.

20. De Filippo C, Cavalieri D, Di Paola M, et al. (2010) Impact of diet in shaping gut microbiota revealed by a comparative study in children from Europe and rural Africa. Proc Natl Acad Sci U S A 107, 14691-14696.

21. Zimmermann MB \& Hurrell RF (2007) Nutritional iron deficiency. Lancet 370, 511-520.

22. WHO (2002) Deficiency Anemia; Assessment, Prevention, and Control; A Guide for Programme Managers. Geneva: World Health Organization.
23. Lynch SR (2011) Why nutritional iron deficiency persists as a worldwide problem. J Nutr 141, 763S-768S.

24. Dostal A, Chassard C, Hilty FM, et al. (2012) Iron depletion and repletion with ferrous sulfate or electrolytic iron modifies the composition and metabolic activity of the gut microbiota in rats. J Nutr 142, 271-277.

25. Werner T, Wagner SJ, Martinez I, et al. (2011) Depletion of luminal iron alters the gut microbiota and prevents Crohn's disease-like ileitis. Gut 60, 325-333.

26. Tompkins GR, O'Dell NL, Bryson IT, et al. (2001) The effects of dietary ferric iron and iron deprivation on the bacterial composition of the mouse intestine. Curr Microbiol 43, 38-42.

27. Dostal A, Fehlbaum S, Chassard C, et al. (2013) Low iron availability in continuous in vitro colonic fermentations induces strong dysbiosis of the child gut microbial consortium and a decrease in main metabolites. FEMS Microbiol Ecol 83, 161-175.

28. Hilty FM, Arnold M, Hilbe M, et al. (2010) Iron from nanocompounds containing iron and zinc is highly bioavailable in rats without tissue accumulation. Nat Nanotechnol $\mathbf{5}$, 374-380.

29. Lee SH, Shinde P, Choi J, et al. (2008) Effects of dietary iron levels on growth performance, hematological status, liver mineral concentration, fecal microflora, and diarrhea incidence in weanling pigs. Biol Trace Elem Res 126, Suppl. 1, S57-S68.

30. Zimmermann MB, Chassard C, Rohner F, et al. (2010) The effects of iron fortification on the gut microbiota in African children: a randomized controlled trial in Cote d'Ivoire. Am J Clin Nutr 92, 1406-1415.

31. Krebs NF, Sherlock LG, Westcott J, et al. (2013) Effects of different complementary feeding regimens on iron status and enteric microbiota in breastfed infants. J Pediatr $\mathbf{1 6 3}$, 416-423.

32. Buhnik-Rosenblau K, Moshe-Belizowski S, Danin-Poleg Y, et al. (2012) Genetic modification of iron metabolism in mice affects the gut microbiota. Biometals 25, 883-892.

33. Winter SE, Thiennimitr P, Winter MG, et al. (2010) Gut inflammation provides a respiratory electron acceptor for Salmonella. Nature 467, 426-429.

34. Winter SE, Lopez CA \& Baumler AJ (2013) The dynamics of gut-associated microbial communities during inflammation. EMBO Rep 14, 319-327.

35. Dethlefsen L, McFall-Ngai M \& Relman DA (2007) An ecological and evolutionary perspective on human-microbe mutualism and disease. Nature 449, 811-818.

36. Alpert C, Sczesny S, Gruhl B, et al. (2008) Long-term stability of the human gut microbiota in two different rat strains. Curr Issues Mol Biol 10, 17-24.

37. Krych L, Hansen CH, Hansen AK, et al. (2013) Quantitatively different, yet qualitatively alike: a meta-analysis of the mouse core gut microbiome with a view towards the human gut microbiome. PLOS ONE 8, e62578.

38. Hanske L, Engst W, Loh G, et al. (2012) Contribution of gut bacteria to the metabolism of cyanidin 3-glucoside in human microbiota-associated rats. Br J Nutr 109, 1433-1441.

39. Wos-Oxley M, Bleich A, Oxley AP, et al. (2012) Comparative evaluation of establishing a human gut microbial community within rodent models. Gut Microbes 3, 234-249.

40. Crouzet L, Gaultier E, Del'homme C, et al. (2013) The hypersensitivity to colonic distension of IBS patients can be transferred to rats through their fecal microbiota. Neurogastroenterol Motil 25, e272-e282.

41. Iannotti LL, Tielsch JM, Black MM, et al. (2006) Iron supplementation in early childhood: health benefits and risks. Am J Clin Nutr 84, 1261-1276. 
42. Forbes AL, Arnaud MJ, Chichester CO, et al. (1989) Comparison of in vitro, animal, and clinical determinations of iron bioavailability: International Nutritional Anemia Consultative Group Task Force report on iron bioavailability. Am J Clin Nutr 49, 225-238.

43. de Sablet T, Chassard C, Bernalier-Donadille A, et al. (2009) Human microbiota-secreted factors inhibit shiga toxin synthesis by enterohemorrhagic Escherichia coli O157:H7. Infect Immun 77, 783-790.

44. Reeves PG, Nielsen FH \& Fahey GC Jr (1993) AIN-93 purified diets for laboratory rodents: final report of the American Institute of Nutrition ad hoc writing committee on the reformulation of the AIN-76A rodent diet. J Nutr 123, 1939-1951.

45. Abatan OI, Welch KB \& Nemzek JA (2008) Evaluation of saphenous venipuncture and modified tail-clip blood collection in mice. J Am Assoc Lab Anim Sci 47, 8-15.

46. Engel MA, Kellermann CA, Rau T, et al. (2008) Ulcerative colitis in AKR mice is attenuated by intraperitoneally administered anandamide. J Physiol Pharmacol 59, 673-689.

47. Zihler A, Gagnon M, Chassard C, et al. (2010) Unexpected consequences of administering bacteriocinogenic probiotic strains for Salmonella populations, revealed by an in vitro colonic model of the child gut. Microbiology 156, 3342-3353.

48. Pereyra LP, Hiibel SR, Prieto Riquelme MV, et al. (2010) Detection and quantification of functional genes of cellulose-degrading, fermentative, and sulfate-reducing bacteria and methanogenic archaea. Appl Environ Microbiol 76, 2192-2202.

49. Ramirez-Farias C, Slezak K, Fuller Z, et al. (2009) Effect of inulin on the human gut microbiota: stimulation of Bifidobacterium adolescentis and Faecalibacterium prausnitzii. Br J Nutr 101, 541-550.

50. Schloss PD, Westcott SL, Ryabin T, et al. (2009) Introducing mothur: open-source, platform-independent, communitysupported software for describing and comparing microbial communities. Appl Environ Microbiol 75, 7537-7541.

51. DeSantis TZ, Hugenholtz P, Larsen N, et al. (2006) Greengenes, a chimera-checked 16S rRNA gene database and workbench compatible with ARB. Appl Environ Microbiol 72, 5069-5072.

52. Edgar RC, Haas BJ, Clemente JC, et al. (2011) UCHIME improves sensitivity and speed of chimera detection. Bioinformatics 27, 2194-2200.

53. Cleusix V, Lacroix C, Vollenweider S, et al. (2008) Glycerol induces reuterin production and decreases Escherichia coli population in an in vitro model of colonic fermentation with immobilized human feces. FEMS Microbiol Ecol 63, 56-64.

54. Gootenberg DB \& Turnbaugh PJ (2011) Companion animals symposium: humanized animal models of the microbiome. J Anim Sci 89, 1531-1537.

55. Kasaoka S, Yamagishi H \& Kitano T (1999) Differences in the effect of iron-deficient diet on tissue weight, hemoglobin concentration and serum triglycerides in Fischer-344, Sprague-Dawley and Wistar rats. J Nutr Sci Vitaminol (Tokyo) 45, 359-366.

56. Lih-Brody L, Powell SR, Collier KP, et al. (1996) Increased oxidative stress and decreased antioxidant defenses in mucosa of inflammatory bowel disease. Dig Dis Sci $\mathbf{4 1}$, 2078-2086.

57. Seril DN, Liao J, Ho KL, et al. (2002) Dietary iron supplementation enhances DSS-induced colitis and associated colorectal carcinoma development in mice. Dig Dis Sci $\mathbf{4 7}$, $1266-1278$

58. Carrier JC, Aghdassi E, Jeejeebhoy K, et al. (2006) Exacerbation of dextran sulfate sodium-induced colitis by dietary iron supplementation: role of NF-kappaB. Int $J$ Colorectal Dis 21, 381-387.
59. Perl DP, Fogarty U, Harpaz N, et al. (2004) Bacterial-metal interactions: the potential role of aluminum and other trace elements in the etiology of Crohn's disease. Inflamm Bowel Dis 10, 881-883.

60. da Silva SM, Venceslau SS, Fernandes CL, et al. (2008) Hydrogen as an energy source for the human pathogen Bilophila wadsworthia. Antonie Van Leeuwenhoek 93, 381-390.

61. Calusinska M, Happe T, Joris B, et al. (2010) The surprising diversity of clostridial hydrogenases: a comparative genomic perspective. Microbiology 156, 1575-1588.

62. Balamurugan R, Mary RR, Chittaranjan S, et al. (2010) Low levels of faecal lactobacilli in women with iron-deficiency anaemia in south India. Br J Nutr 104, 931-934.

63. Cuiv PO, Klaassens ES, Durkin AS, et al. (2011) Draft genome sequence of Turicibacter sanguinis PC909, isolated from human feces. J Bacteriol 193, 1288-1289.

64. Arias CA \& Murray BE (2012) The rise of the Enterococcus: beyond vancomycin resistance. Nat Rev Microbiol 10, $266-278$

65. Louis P, Young P, Holtrop G, et al. (2010) Diversity of human colonic butyrate-producing bacteria revealed by analysis of the butyryl-CoA:acetate CoA-transferase gene. Environ Microbiol 12, 304-314.

66. Karadag D \& Puhakka JA (2010) Enhancement of anaerobic hydrogen production by iron and nickel. Int J Hydrogen Energy 35, 8554-8560.

67. Wei H, Dong L, Wang T, et al. (2010) Structural shifts of gut microbiota as surrogate endpoints for monitoring host health changes induced by carcinogen exposure. FEMS Microbiol Ecol 73, 577-586.

68. Conterno L, Fava F, Viola R, et al. (2011) Obesity and the gut microbiota: does up-regulating colonic fermentation protect against obesity and metabolic disease? Genes Nutr 6, 241-260.

69. Hosseini E, Grootaert C, Verstraete W, et al. (2011) Propionate as a health-promoting microbial metabolite in the human gut. Nutr Rev 69, 245-258.

70. Luhrs H, Kudlich T, Neumann M, et al. (2002) Butyrateenhanced TNFalpha-induced apoptosis is associated with inhibition of NF-kappaB. Anticancer Res 22, 1561-1568.

71. Luhrs H, Gerke T, Muller JG, et al. (2002) Butyrate inhibits NF-kappaB activation in lamina propria macrophages of patients with ulcerative colitis. Scand J Gastroenterol 37, 458-466.

72. Roy MJ, Dionne S, Marx G, et al. (2009) In vitro studies on the inhibition of colon cancer by butyrate and carnitine. Nutrition 25, 1193-1201.

73. Chassard C, Dapoigny M, Scott KP, et al. (2012) Functional dysbiosis within the gut microbiota of patients with constipated-irritable bowel syndrome. Aliment Pharmacol Ther 35, 828-838.

74. Carbonero F, Benefiel AC, Alizadeh-Ghamsari AH, et al. (2012) Microbial pathways in colonic sulfur metabolism and links with health and disease. Front Physiol 3, 448.

75. Marquet P, Duncan SH, Chassard C, et al. (2009) Lactate has the potential to promote hydrogen sulphide formation in the human colon. FEMS Microbiol Lett 299, 128-134.

76. Attene-Ramos MS, Nava GM, Muellner MG, et al. (2010) DNA damage and toxicogenomic analyses of hydrogen sulfide in human intestinal epithelial FHs 74 Int cells. Environ Mol Mutagen 51, 304-314.

77. Wallace JL, Vong L, McKnight W, et al. (2009) Endogenous and exogenous hydrogen sulfide promotes resolution of colitis in rats. Gastroenterology 137, 569-578, 578 e 561.

78. Hirata I, Naito Y, Takagi T, et al. (2011) Endogenous hydrogen sulfide is an anti-inflammatory molecule in dextran sodium sulfate-induced colitis in mice. Dig Dis Sci 56, 1379-1386. 